\title{
Asynchronous Random Sleeping for Sensor Networks
}

\author{
CUNQING HUA and TAK-SHING PETER YUM \\ The Chinese University of Hong Kong
}

Sleeping scheduling is a common energy-conservation solution for sensor networks. For application whereby coordination of sleeping among sensors is not possible or inconvenient, random sleeping is the only option. In this article, we study the asynchronous random sleeping(ARS) scheme whereby sensors (i) do not need to synchronize with each other, and (ii) do not need to coordinate their sleeping schedules. The stationary coverage probability and the expected coverage periods for ARS are derived. For surveillance application, we derive in addition the detection probability and detection delay distribution. The correctness of our results is validated through extensive simulations. We compare ARS with other synchronous and asynchronous sleeping scheduling algorithms and show that ARS offers better performance in terms of detection delay in the lower duty-cycle regime. We also conduct simulations to demonstrate that our results can be a good approximation for clock drifting case.

Categories and Subject Descriptors: C.2.2 [Computer-Communication Networks]: Network Protocols-Applications (SMTP, FTP, etc.); G.2 [Discrete Mathematics]: Probability and Statistics-Stochastic processes

General Terms: Algorithms, Theory, Performance

Additional Key Words and Phrases: Sensor network, random sleeping, energy conservation, $k$-coverage, event detection

ACM Reference Format:

Hua, C. and Yum, T.-S. P. 2007. Asynchronous random sleeping for sensor networks. ACM Trans. Sens. Netw. 3, 3, Article 15 (August 2007), 25 pages. DOI $=10.1145 / 1267060.1267063$ http://doi.acm.org/10.1145/1267060.1267063

\section{INTRODUCTION}

The design of energy-efficient protocols is one of critical issues for wireless sensor networks. This is especially important for the applications where the sensor

This work was supported in part by the Hong Kong Research Grants Council under Grant CUHK 4220/03E.

Authors' current addresses: C. Hua, The University of Houston, 311 Philip G. Hoffman Hall, 4800 Calhoun Rd., Houston, TX 77204-3010; email: cqhua@cs.uh.edu; T.-S. P. Yum, Information Engineering Department, The Chinese University of Hong Kong, Shatin, N. T., Hong Kong. email: yum@ie. cuhk. edu.hk.

Permission to make digital or hard copies of part or all of this work for personal or classroom use is granted without fee provided that copies are not made or distributed for profit or direct commercial advantage and that copies show this notice on the first page or initial screen of a display along with the full citation. Copyrights for components of this work owned by others than ACM must be honored. Abstracting with credit is permitted. To copy otherwise, to republish, to post on servers, to redistribute to lists, or to use any component of this work in other works requires prior specific permission and/or a fee. Permissions may be requested from Publications Dept., ACM, Inc., 2 Penn Plaza, Suite 701, New York, NY 10121-0701 USA, fax +1 (212) 869-0481, or permissions@acm.org. (C) 2007 ACM 1550-4859/2007/08-ART15 \$5.00 DOI 10.1145/1267060.1267063 http://doi.acm.org/ $10.1145 / 1267060.1267063$ 
network has to be deployed unattended over a long period without replacing the batteries. Different solutions have been proposed to reduce the energy consumption of sensor network, such as reducing communication range, reducing data traffic through in-network data compression, balancing traffic with maximum lifetime routing, etc. In this article, we investigate a class of power conserving techniques through active/sleeping planning. The basic idea is to schedule the duty cycles of sensor nodes such that off-duty sensors are turned off as long as the network functionality can be maintained by other active nodes. Offduty sensors have power consumption only around $0.1 \%$ of that in active mode [Crossbow 2004; Kumar et al. 2004].

Existing sleeping schemes for sensor networks fall into two categories: the coordinated sleeping mechanism and the random sleeping mechanism. In coordinated sleeping mechanisms, sensor nodes communicate with each other to adjust sleeping schedules to satisfy some quality of service constraints such as coverage, connectivity, data delivery ratio, etc.. For example, in order to avoid losing sensing coverage, a sleeping scheduling mechanism was proposed in Tian and Georganas [2002] that allows a sensor to shut down only if its sensing area is completely covered by its neighbors, which is done by checking the sponsored sectors according to the location and sensing radius of other working neighbors. In Zhang and Hou [2004a], an optimal geographic density control (OGDC) scheme was proposed to ensure 1-coverage and 1-connectivity. The basic idea of OGDC is to minimize the overlapping area of active sensors so that more sensors can be turned off. A sensor is turned on only if it minimizes the overlapping area with the working sensors and covers a crossing point of two active sensors. In Wang et al. [2003], an integrated coverage and connectivity configuration protocol(CCP) was proposed to maximize the number of sleeping nodes while guaranteeing both $k$-coverage and $k$-connectivity of the network. A sensor enters sleeping mode only when its coverage area is k-covered by all its neighbors, which can be verified by checking whether all crossing points inside its coverage area are $k$-covered. The $k$-coverage problem was also studied in Yan et al. [2003], which tries to provide differentiated surveillance service by coordinating the sleeping and wakeup time among neighboring nodes. In [Cao et al. 2005a], an optimal coordinated sleep was proposed to guarantee a bounded-delay sensing coverage and maximize the network lifetime. A node can calculate its wakeup time based on its neighbors' schedules. This process is repeated in iterations and eventually all nodes can reach a local scheduling decision. Some other sleeping schemes assume that the sensors are organized into clusters [Li et al. 2003; He et al. 2004; Moscibroda and Wattenhofer 2005; Deng et al. 2005]. Each cluster has a cluster head to manage the communication between nearby nodes and the base station. A comprehensive survey on coordinated sleeping schemes can be found in Wang and Xiao [2005].

In random sleeping mechanisms, sensors decide to enter sleeping mode randomly and independent of others. For example, the Randomized Independent Sleeping (RIS) mechanism [Kumar et al. 2004] assumes that sensors are synchronized globally and time is divided into slots. At the beginning of each time slot, a sensor independently decides to remain awake or enter sleeping mode with probability of $p$ and $1-p$, respectively. Therefore, the duration of 
active/sleeping periods are geometrically distributed. A variation of RIS was proposed in Gui and Mohapatra [2004], where each time slot is divided into two parts - the active period of length $p T$ and the sleeping period of length $(1-p) T$, where $T$ is the length of the duty cycle.

Random sleeping mechanism cannot ensure complete coverage all the time, so it is important to investigate coverage characteristics under different conditions. Kumar et al. [2004], derived the critical conditions for asymptotic k-coverage of RIS mechanism under three different sensor placement mechanisms-grid, random uniform and two-dimensional Poisson. Shakkottai et al. [2003], derived the necessary and sufficient conditions for the 1-coverage and 1-connectivity for the grid network, where $n$ sensors are deployed over a $\sqrt{n} \times \sqrt{n}$ grid. These results suggest that, if node density is higher enough, the connectivity and coverage can be guaranteed even if the sensor nodes are highly unreliable and the transmission range small. In Hsin and Liu [2004], the coverage extensity (the probability that a given location is not covered) and coverage intensity (the tail distribution of a location not covered for longer than a given period of time) were examined for a randomized sleeping scheme. Zhang and Hou [2004b] derived the necessary and sufficient conditions of the node density in order to maintain complete $k$-coverage. These analytical results on coverage probability were based on the assumption that all sensors are synchronized.

Most of these coordinated and random sleeping schemes require that the time are synchronized among sensors, which are nontrivial issues for large sensor networks [Aspnes et al. 2004; Elson et al. 2002]. To circumvent such difficulty, some asynchronous sleeping schemes have been proposed [Zheng et al. 2003; Paruchuri et al. 2004]. However, in this case, it is a challenging problem to find the coverage and noncoverage periods of a location, which is important for some applications such as surveillance services [Gui and Mohapatra 2004; Cao et al. $2005 \mathrm{~b}$ ] where the intrusion detection capability of the system is characterized by these metrics. For example, Cao et al. [2005b] derived the distribution of detection delay for both stationary and mobile targets assuming that a special asynchronous sleeping scheme as in Gui and Mohapatra [2004] are used, in which all sensors wake up randomly and remain awake for a certain time period in each duty cycle.

In this article, we study a different asynchronous random sleeping (ARS) mechanism for sensor networks, whereby sensors wake up randomly and independent of others in each time slot. We assume that sensors are not synchronized and the offsets of the time slot between nodes are uniformed distributed. We derive the closed-form expressions of the stationary coverage probability and the expected $k$-coverage periods, with which we obtain the expected k-vulnerable periods using the alternating renewal process theory. For the application of ARS in the surveillance system, we derive the closed-form expressions of the detection probability and detection delay distribution. We provide numerical and simulation results to demonstrate the performance of ARS and compare it with other synchronous and asynchronous random sleeping scheduling schemes.

Our analytical results have a wide range of applications. A simple example is the grid deployment model where sensors are deployed in regular grid over 
the field [Kumar et al. 2004]. In this case, the analytical results for a specific point are applicable for overall network. Our results therefore can be used to decide the parameters according to the application requirements. For example, we can find the corresponding node density and wakeup probability for certain $k$-coverage requirement. For random uniform deployment or Poisson deployment, it is straightforward to extend our results with the consideration of the sensor node distribution.

In the following, we first introduce the system models in Section 2 . We then derive the expected coverage periods for various coverage degrees in Section 3 and the expected vulnerable periods in Section 4 . The surveillance application of ARS is studied in Section 5 and the detection probability and detection delay distribution are derived. Numerical and simulation results are presented in Section 6 and finally we conclude this article in Section 7. In the Appendix, we show the derivation of mathematical functions important to our research.

\section{SYSTEM MODELS}

We consider the Boolean sensing model, which has been widely adopted in the study of sensor networks [Tian and Georganas 2002; Shakkottai et al. 2003; Liu and Towsley 2004]. In the Boolean sensing model, the sensing area of each sensor is a disc with certain radius $r$, and a location is detectable only if it lies within the sensing areas of some sensors. We consider a certain location covered by a set of $n$ nodes labeled with $0,1, \ldots, n-1$, where $n$ is a random variable that can be determined by the sensor placement models such as that in Kumar et al. [2004]. We assume that these sensors are not synchronized and the time of each sensor is divided into slots of fixed length of one unit. Sensors independently decide whether to awake up in a time slot (with probability $p$ ) or remain sleeping (with probability $1-p$ ). For nonregular sensing models or a general sensing model such as that in Tian and Georganas [2002], all subsequent results are still applicable as long as the number of covering sensors is known; however, we use Boolean sensing model in this article for simplicity.

Without loss of generality, we assume that the time slot of node $i, i=$ $1, \ldots, n-1$ has an offset $U_{i}$ with respect to node 0 , which are i.i.d. random variables in $(0,1)$. Therefore, the probability density function of $U_{i}$ is simply given by

$$
f_{i}\left(x_{i}\right)=\left\{\begin{array}{l}
1,0<x_{i}<1, i=1, \cdots, n-1 \\
0, \text { elsewhere }
\end{array}\right.
$$

The joint density of $U_{1}, \ldots, U_{n-1}$ is the product of the marginal densities. That is,

$$
f\left(x_{1}, \ldots, x_{n-1}\right)=1, \quad 0<x_{i}<1, i=1, \ldots, n-1
$$

and zero elsewhere.

Let $V_{i}$ denote the $i$ th smallest of these random variables; then $V_{1}, \ldots, V_{n-1}$ are ordered statistics and their joint probability density function is well known to be Mathai [1999]

$$
g\left(y_{1}, \ldots, y_{n-1}\right)=(n-1) !, \quad 0<y_{1}<\cdots<y_{n-1}<1,
$$

and zero elsewhere. 


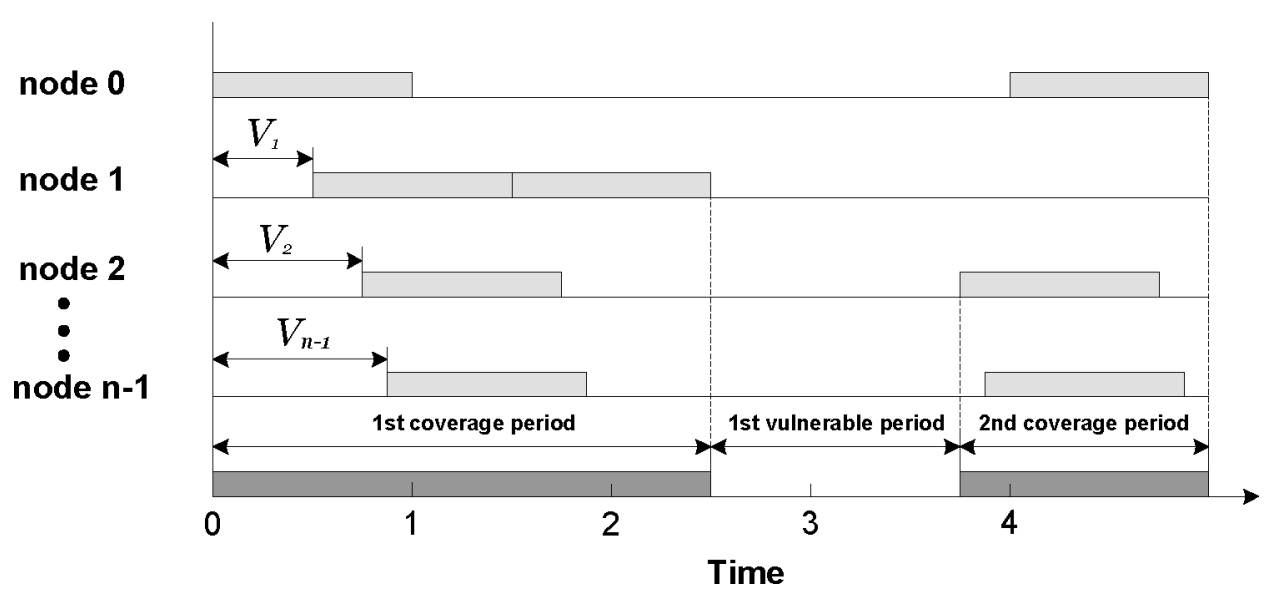

Fig. 1. Lengths of coverage and vulnerable periods.

The probability density function of $V_{i}$ can be derived by integrating over $g\left(y_{1}, \ldots, y_{n-1}\right)$ as

$$
\begin{aligned}
h_{i}(y) & =\int \cdots \int_{\Omega} g\left(y_{1}, \ldots, y_{n-1}\right) d y_{1} \cdots d y_{n-1} \\
& =\frac{\Gamma(n)}{\Gamma(n-i) \Gamma(i)} y^{i-1}(1-y)^{n-1-i}, i=1, \ldots, n-1,
\end{aligned}
$$

where $\Omega=\left\{\left(y_{1}, \ldots, y_{i-1}, y_{i+1}, \ldots, y_{n-1}\right) \mid 0<y_{1} \ldots<y_{i-1}<y<y_{i+1} \cdots<\right.$ $y_{n-1}<1$ \}. Clearly, $h_{i}(y)$ is a beta density function with parameters $(i, n-i)$, and the first moment of $V_{i}$ is $E\left[V_{i}\right]=i / n$.

\section{ANALYSIS OF COVERAGE PERIODS}

Asynchronous random sleeping (ARS) can only offer statistical sensing coverage; its performance can be characterized by the stationary coverage probability and the coverage periods. In this section, we derive the distribution of $k$-coverage periods for ARS. By $k$-coverage we mean the monitored location is covered by at least $k$ active nodes, or the coverage degree is $k$ or above. Therefore, a $k$-coverage period is the time duration that the location is covered by at least $k$ nodes. A $k$-coverage period ends at the instant that the number of active nodes drops below $k$; then a $k$-vulnerable period starts. In this fashion, the coverage periods alternate with vulnerable periods, as shown in Figure 1 for $k=1$. This can be modeled as an alternating renewal process with two states. Let the random variables $Y_{1}^{(k)}, Y_{2}^{(k)}, \ldots$ and $Z_{1}^{(k)}, Z_{2}^{(k)}, \ldots$ denote lengths of successive $k$-coverage and $k$-vulnerable periods, respectively. By alternating renewal theory, the sequences of random variables $\left\{Y_{i}^{(k)}\right\}$ and $\left\{Z_{i}^{(k)}\right\}$ are independent and identically distributed. Let $E\left[Y^{(k)}\right]=E\left[Y_{i}^{(k)}\right]$ and $E\left[Z^{(k)}\right]=E\left[Z_{i}^{(k)}\right]$ denote the mean lengths of $k$-coverage and $k$-vulnerable periods, respectively, which will be derived in the following subsections. 
$\begin{array}{lll}\text { Article } 15 / 6 & \text { C } & \text { C. Q. Hua and T.-S. Yum }\end{array}$

\subsection{1-Coverage Periods}

An 1-coverage period is started when a node wakes up to find no other nodes are active. Consider a specific coverage period initiated by node 0 when it wakes up at time $t_{0}$; this coverage period lasts for at least one time slot up to time $t_{0}+1$, during which node $1, \ldots, n-1$ may wake up. Therefore:

(i) If none of the nodes $1,2, \ldots, n-1$ wakes up in $\left(t_{0}, t_{0}+1\right)$, and node 0 goes to sleep in the next time slot, then this 1-coverage period of length 1 ends at time $t_{0}+1$. This happens with probability $(1-p)^{n}$.

(ii) If none of the nodes $1,2, \ldots, n-1$ wakes up in $\left(t_{0}, t_{0}+1\right)$, but node 0 remains awake in the next time slot, then this 1-coverage period is renewed at time $t_{0}+1$ by node 0 . This happens with probability $p(1-p)^{n-1}$.

(iii) If at least one of the nodes $1,2, \ldots, n-1$ wakes up in $\left(t_{0}, t_{0}+1\right)$, and node $j(1 \leq j \leq n-1)$ is the first such node, then this 1-coverage period is renewed at time $t_{0}+V_{j}$ by node $j$. This happens with probability $p(1-p)^{j-1}$.

Let us define the 1-coverage period distribution as $G(y)$ :

$$
G(y) \triangleq P\left\{Y^{(1)} \leq y\right\} .
$$

Let $Y_{j}^{(1)}$ denote the 1-coverage period started by node $j$. Under the condition that the relative time slot offsets are uniform distributed, all $Y_{j}^{(1)}$ 's are i.i.d. random variables distributed as $G(y)$. We next derive the Laplace transform of the pdf associated with $Y^{(1)}$, which is given by

$$
G^{*}(s) \triangleq \int_{0}^{\infty} e^{-s y} d G(y)
$$

which can also be expressed in the expectation form as $G^{*}(s) \triangleq E\left[e^{-s y}\right]$.

Conditioning on the three events above, we have

$$
\begin{aligned}
E\left[e^{-s Y^{(1)}}\right]= & (1-p)^{n} E\left[e^{-s \cdot 1}\right]+p(1-p)^{n-1} E\left[e^{-s\left(1+Y_{0}^{(1)}\right)}\right] \\
& +\sum_{j=1}^{n-1} p(1-p)^{j-1} E\left[e^{-s\left(V_{j}+Y_{j}^{(1)}\right)}\right] \\
= & (1-p)^{n} E\left[e^{-s}\right]+p(1-p)^{n-1} E\left[e^{-s}\right] E\left[e^{\left.-s Y_{0}^{(1)}\right)}\right] \\
& +\sum_{j=1}^{n-1} p(1-p)^{j-1} E\left[e^{-s V_{j}}\right] E\left[e^{-s Y_{j}^{(1)}}\right],
\end{aligned}
$$

Let $B_{j}^{*}(s)=E\left[e^{-s V_{j}}\right]$ denote the Laplace transform of the distribution function of $V_{j}$. Since all $Y_{j}^{(1)}$ have the identical distribution with corresponding transforms $G^{*}(s)$, we have

$$
G^{*}(s)=(1-p)^{n} e^{-s}+p(1-p)^{n-1} e^{-s} G^{*}(s)+\sum_{j=1}^{n-1} p(1-p)^{j-1} B_{j}^{*}(s) G^{*}(s) .
$$


Solving for $G^{*}(s)$, we have

$$
G^{*}(s)=\frac{(1-p)^{n} e^{-s}}{1-p(1-p)^{n-1} e^{-s}-\sum_{j=1}^{n-1} p(1-p)^{j-1} B_{j}^{*}(s)},
$$

which can be further simplified by defining $B^{*}(s)=\sum_{j=1}^{n-1} p(1-p)^{j-1} B_{j}^{*}(s) ; B^{*}(s)$ is derived in Appendix A.

We can obtain the expectation of $Y^{(1)}$ from (5) with $E\left[Y^{(1)}\right]=-G^{*^{\prime}}(0)$, which, however, is nontrivial. Instead, we can obtain a recursive formula for $E\left[Y_{0}^{(1)}\right]$ conditioning on the three events above:

$$
\begin{aligned}
E\left[Y_{0}^{(1)}\right]= & (1-p)^{n} \cdot 1+p(1-p)^{n-1}\left(1+E\left[Y_{0}^{(1)}\right]\right) \\
& +\sum_{j=1}^{n-1} p(1-p)^{j-1}\left(E\left[V_{j}\right]+E\left[Y_{j}^{(1)}\right]\right) .
\end{aligned}
$$

Since all $E\left[Y_{j}^{(1)}\right]$ 's are equal, we denote them as $E\left[Y^{(1)}\right]$. Substituting $E\left[V_{j}\right]=$ $j / n$ into (6), rewriting the second term as $p(1-p)^{n}\left(n / n+E\left[Y^{(1)}\right]\right)$, and merging it with the third term, we can simplify (6) as

$$
\begin{aligned}
E\left[Y^{(1)}\right] & =(1-p)^{n}+\sum_{j=1}^{n} p(1-p)^{j-1}\left(\frac{j}{n}+E\left[Y^{(1)}\right]\right) \\
& =(1-p)^{n}+\frac{p}{n} \sum_{j=1}^{n} j(1-p)^{j-1}+E\left[Y^{(1)}\right] \sum_{j=1}^{n}(1-p)^{j-1} \\
& =(1-p)^{n}+\frac{1-(n p+1)(1-p)^{n}}{n p}+\left(1-(1-p)^{n}\right) E\left[Y^{(1)}\right] \\
& =\frac{1-(1-p)^{n}}{n p}+\left(1-(1-p)^{n}\right) E\left[Y^{(1)}\right] .
\end{aligned}
$$

Solving for $E\left[Y^{(1)}\right]$, we obtain

$$
E\left[Y^{(1)}\right]=\frac{1-(1-p)^{n}}{n p(1-p)^{n}}
$$

\subsection{2-Coverage Periods}

A 2-coverage period starts when a node wakes up and finds that there is exactly one other active node. Let $Y_{i}^{(2)}$ denote a specific 2-coverage period initiated by node 0 when it wakes up at time $t_{0}$ and finds node $i$ be the only active node, as shown in Figure 2. This 2-coverage period is guaranteed to last up to $t_{0}+V_{i}$ (the residual life of node $i$ ), during which only node $1, \ldots, i-1$ may wake up. Therefore:

(i) If none of the nodes $1, \ldots, i-1$ wakes up in $\left(t_{0}, t_{0}+v_{i}\right)$ and node $i$ goes to sleep at its next time slot, this 2-coverage period of length $V_{i}$ ends at time $t_{0}+V_{i}$. This happens with probability $(1-p)^{i}$.

(ii) If at least one of the nodes $1, \ldots, i-1$ wakes up in $\left(t_{0}, t_{0}+V_{i}\right)$, or node $i$ remains awake at the next time slot; suppose that node $j(1 \leq j \leq i)$ is the first wakeup node. Then the 2-coverage period is renewed by node $j$ at 


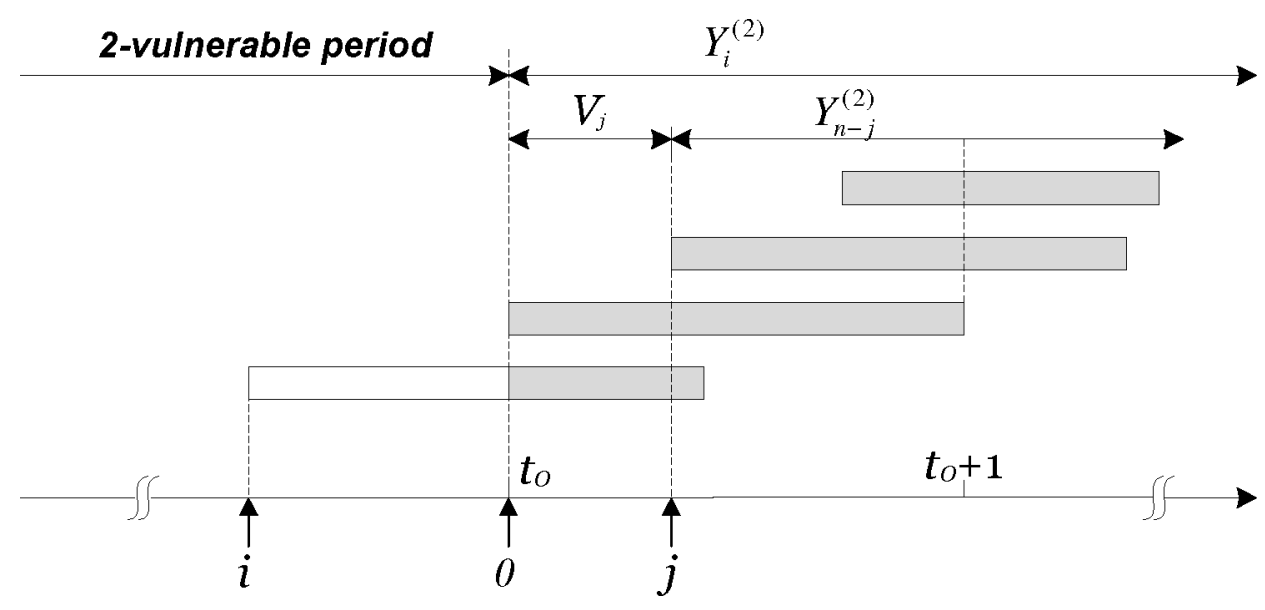

Fig. 2. $Y_{i}^{(2)}$ : the 2-coverage period initiated by node $i$ and 0 .

time $t_{0}+V_{j}$. The new 2-coverage period is provided by node 0 and $j$, so it is guaranteed to last up to the end of node 0 's active slot at time $t_{0}+1$. During this new 2 -coverage period, only node $j+1, \ldots, n-1,0$ may wake up, so it can be presented by $Y_{n-j}^{(2)}$, as shown in Figure 2 . This happens with probability $p(1-p)^{j-1}$.

It is nontrivial to derive the closed-form expression for the Laplace transform of $Y^{(2)}$ as that in previous section. Therefore, we focus on the derivation of its expectation. Notice that a recursive formula for $E\left[Y_{i}^{(2)}\right]$ can be derived conditioning on the two events above as

$$
\begin{aligned}
E\left[Y_{i}^{(2)}\right] & =(1-p)^{i} E\left[V_{i}\right]+\sum_{j=1}^{i} p(1-p)^{j-1}\left(E\left[V_{j}\right]+E\left[Y_{n-j}^{(2)}\right]\right) \\
& =\frac{i(1-p)^{i}}{n}+\frac{1-(i p+1-p)(1-p)^{i}}{n p}+\sum_{j=1}^{i} p(1-p)^{j-1} E\left[Y_{n-j}^{(2)}\right] \\
& =\frac{1-(1-p)^{i}}{n p}+\sum_{j=1}^{i} p(1-p)^{j-1} E\left[Y_{n-j}^{(2)}\right], \quad i=1, \ldots, n-1 .
\end{aligned}
$$

Note when node 0 wakes up and finds only one node is active, that node can be any of the nodes in $1, \ldots, n-1$. In this case, a new 2 -coverage period starts; Or that node can be node 0 if it remains awake for two consecutive time slots. However, this case does not lead to a new 2-coverage period. Since these $n$ events occur equally, we have

$$
E\left[Y^{(2)}\right]=\frac{1}{n} \sum_{i=1}^{n-1} E\left[Y_{i}^{(2)}\right]
$$

Solving the set of $n-1$ linear equations in (8), we can find individual $E\left[Y_{i}^{(2)}\right]$ 's, with which we can obtain $E\left[Y^{(2)}\right]$ from (9). However, we show in Appendix B 


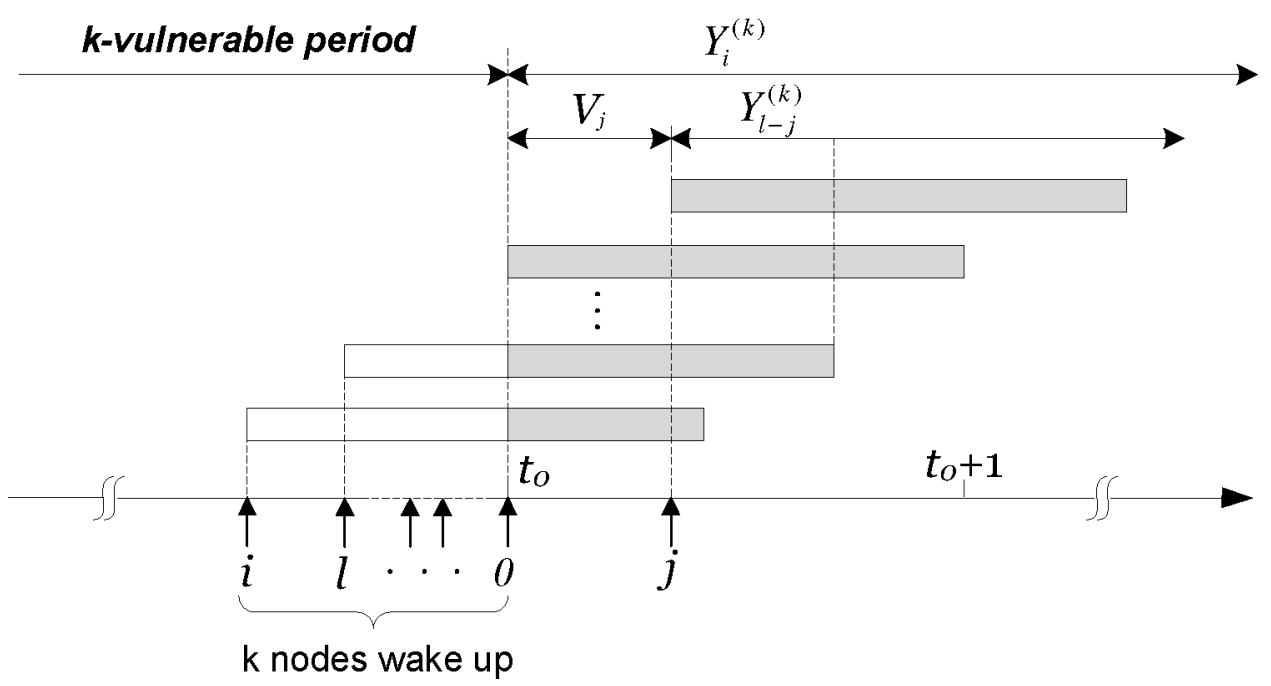

Fig. 3. $Y_{i}^{(k)}$ : the $k$-coverage period initiated by node $i$ and 0 .

that, without explicitly solving for individual $E\left[Y_{i}^{(2)}\right]$ 's, we can derive the closedform expression of $E\left[Y^{(2)}\right]$ as

$$
E\left[Y^{(2)}\right]=\frac{1}{n} \sum_{i=1}^{n-1} E\left[Y_{i}^{(2)}\right]=\frac{1-(1-p)^{n}-n p(1-p)^{n-1}}{n\left(\begin{array}{l}
n \\
1
\end{array}\right) p^{2}(1-p)^{n-1}} .
$$

\section{3 $k$-Coverage Periods $(k \geq 3)$}

For general $k$ that equals 3 and above, a $k$-coverage period is started when a node wakes up and finds that exact $k-1$ other nodes are active. Suppose that node 0 wakes up at time $t_{0}$ and finds other $k-1$ active nodes are active, and node $i$ is the first one of them (i.e., node $i$ is the earliest wakeup node among these $k-1$ nodes); then a $k$-coverage period is initiated by node 0 with node $i$ and other $k-2$ nodes, which we denote by $Y_{i}^{(k)}$ as shown in Figure 3. The length of this $k$-coverage period is the residual life of node $i$, during which only node $1, \ldots, i-1$ may wake up. Therefore:

(i) If none of the nodes $1, \cdots, i-1$ wakes up in $\left(t_{0}, t_{0}+V_{i}\right)$ and node $i$ goes to sleep at its next time slot, then this $k$-coverage period of length $V_{i}$ ends at time $t_{0}+V_{i}$. This happens with probability $(1-p)^{i}$.

(ii) If at least one of the nodes $1, \ldots, i-1$ wakes up in $\left(t_{0}, t_{0}+v_{i}\right)$, or node $i$ remains awake at its next time slot, suppose that node $j(1 \leq j \leq i)$ is the first wakeup nodes. Then the $k$-coverage period is renewed by node $j$ at time $t_{0}+V_{j}$. This new $k$-coverage period is extended to the end of the active slot of node $l$, which is the first wakeup node after node $i$ among these $k-2$ active nods. This new k-coverage period can be denoted by $Y_{l-j}^{(k)}$, as shown in Figure 3. 
Since any $k-2$ nodes in $i+1, \ldots, n-1$ can be the active nodes, the probability that node $l$ being the first one is given by

$$
P_{l}=\frac{\left(\begin{array}{c}
n-1-l \\
k-3
\end{array}\right)}{\left(\begin{array}{c}
n-1-i \\
k-2
\end{array}\right)}, \quad l=i+1, \ldots, n+2-k .
$$

Therefore, a recursive formula for $E\left[Y_{i}^{(k)}\right]$ can be derived conditioning on node $j$ and $l$ as

$$
\begin{aligned}
E\left[Y_{i}^{(k)}\right]= & (1-p)^{i} E\left[V_{i}\right]+\sum_{j=1}^{i} p(1-p)^{i-1}\left(E\left[V_{j}\right]+\sum_{l=i+1}^{n+2-k} \frac{\left(\begin{array}{c}
n-1-l \\
k-3
\end{array}\right)}{\left(\begin{array}{c}
n-1-i \\
k-2
\end{array}\right)} E\left[Y_{l-j}^{(k)}\right]\right) \\
= & \frac{1-(1-p)^{i}}{n p}+\sum_{j=1}^{i} p(1-p)^{i-1} \\
& \times \sum_{l=i+1}^{n-1} \frac{\left(\begin{array}{c}
n-1-l \\
k-3
\end{array}\right)}{\left(\begin{array}{c}
n-1-i \\
k-2
\end{array}\right)} E\left[Y_{l-j}^{(k)}\right], i=1, \ldots, n+1-k
\end{aligned}
$$

Since it is equally likely for node 0 to initiate a $k$-coverage period with any $k-1$ nodes in $0,1, \ldots, n-1$, the possible number of combinations is $\left(\begin{array}{c}n \\ k-1\end{array}\right)$. Among them, the number of combinations for node $i$ to be the first active node is just $\left(\begin{array}{c}n-1-i \\ k-2\end{array}\right)$. Therefore

$$
E\left[Y^{(k)}\right]=\sum_{i=1}^{n+1-k} \frac{\left(\begin{array}{c}
n-1-i \\
k-2
\end{array}\right)}{\left(\begin{array}{c}
n \\
k-1
\end{array}\right)} E\left[Y_{i}^{(k)}\right]
$$

Solving the set of linear equations of (12), we can find individual $E\left[Y_{i}^{(k)}\right]$, with which we can obtain $E\left[Y^{(k)}\right]$ from (13). But from the closed-form expressions of $E\left[Y^{(1)}\right]$ and $E\left[Y^{(2)}\right]$ in (7) and (10), we can infer a closed-form expression for $E\left[Y^{(k)}\right]$ as follows:

$$
E\left[Y^{(k)}\right]=\frac{1-\sum_{i=0}^{k-1}\left(\begin{array}{c}
n \\
i
\end{array}\right) p^{i}(1-p)^{n-i}}{n\left(\begin{array}{c}
n \\
k-1
\end{array}\right) p^{k}(1-p)^{n-k+1}}, \quad k=1, \ldots, n .
$$

This solution is verified to be identical to that obtained from (13) for all $n$ and $k(n \geq k)$.

\section{EXPECTED VULNERABLE PERIODS}

It is easy to see from Figure 1 that the asynchronous sleeping mechanism does not change the coverage intensity. That is, in the stationary state, the number of active sensors at a specific time instant follows a binomial distribution like that of the synchronous sleeping scheme. Therefore, let $P^{(k)}$ denote the $k$-coverage probability, and we have

$$
P^{(k)}=1-\sum_{i=0}^{k-1}\left(\begin{array}{c}
n \\
i
\end{array}\right) p^{i}(1-p)^{n-i}
$$


Let $I(k, t)$ be an indicator variable

$$
I(k, t)=\left\{\begin{array}{l}
1, \text { the location is } k \text {-covered at time } \mathrm{t} ; \\
0, \text { otherwise. }
\end{array}\right.
$$

By the alternating renewal process theory[Ross 1996], the stationary $k$-coverage probability is given by

$$
P^{(k)}=\lim _{t \rightarrow \infty} P(I(k, t)=1)=\frac{E\left[Y^{(k)}\right]}{E\left[Y^{(k)}\right]+E\left[Z^{(k)}\right]} .
$$

Equating (15) and (16), we see that

$$
E\left[Z^{(k)}\right]=\frac{\sum_{i=0}^{k-1}\left(\begin{array}{c}
n \\
i
\end{array}\right) p^{i}(1-p)^{n-i}}{1-\sum_{i=0}^{k-1}\left(\begin{array}{l}
n \\
i
\end{array}\right) p^{i}(1-p)^{n-i}} E\left[Y^{(k)}\right] .
$$

From (17) and (14) we can obtain the expected vulnerable periods $E\left[Z^{(k)}\right]$ as

$$
E\left[Z^{(k)}\right]=\frac{\sum_{i=0}^{k-1}\left(\begin{array}{l}
n \\
i
\end{array}\right) p^{i}(1-p)^{n-i}}{n\left(\begin{array}{c}
n \\
k-1
\end{array}\right) p^{k}(1-p)^{n-k+1}}, \quad k=1, \ldots, n .
$$

\section{SURVEILLANCE APPLICATION}

One application of ARS is in surveillance-type sensor networks. In surveillance application, events occur infrequently. Therefore, to minimize power consumption while guaranteeing a certain level of vigilance, sensors are typically operated in three modes-sleeping mode, wakeup mode, and tracking mode. The tracking mode differs from wakeup mode in that a sensor in tracking mode should remain active until the end of the event, while a sensor in wakeup mode can switch to sleeping mode at the end of a time slot. Since events can always be detected when a sensor is in the tracking mode, we focus on the detection performance during the sleeping/wakeup modes when ARS is used. The events considered in our study are stationary objects with stochastic durations (such as a wildfire). However, the analysis techniques in Cao et al. [2005b] can be extended to analyze the performance of ARS for mobile objects, which, however, is beyond the scope of this article.

\subsection{Single Sensor}

We first study the basic case where the target region is monitored by a single sensor. The analysis of this single sensor case will be generalized to the multiple sensor case in the next subsection.

Without loss of generality, let the occurrence time of events be a Poisson process. Consider an event occurring during the sleeping mode of the sensor at time $t=0$, as shown in Figure 4 . Let $W$ denote the wakeup delay. It consists of two parts:

(i) The random time offset $S$ from the occurrence of the event to the boundary of the sensor's duty cycle, which is uniformly distributed in $(0,1)$ under the Poisson event occurrence assumption. 
$\begin{array}{lll}\text { Article } 15 / 12 & \text { • C. Q. Hua and T.-S. Yum }\end{array}$

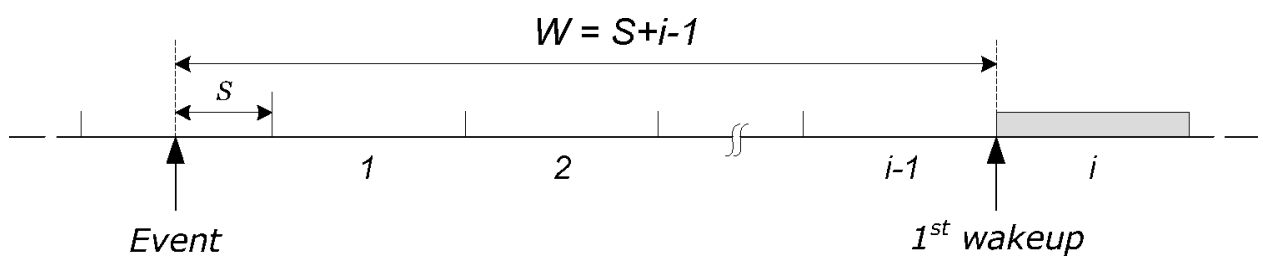

Fig. 4. Wakeup delay with a single sensor.

(ii) The remaining time $R$ (in whole number of slots) until the sensor's first wakeup. It is geometrically distributed as $P\{R=i\}=p(1-p)^{i}, i=1,2, \ldots$

Therefore, given $R=i$, the wakeup delay $W=S+i-1$ is uniformly distributed in $(i-1, i)$.

Let $r$ be the integer part of $t, f_{W}(t)$ be the probability density function (PDF) of $W$, and $F_{W}(t)$ be the corresponding distribution function. Removing the conditioning on $R$, we have

$$
f_{W}(t)=\sum_{i=0}^{\infty} f_{W}(t \mid R=i) P\{R=i\}=p(1-p)^{r+1}
$$

and

$$
F_{W}(t)=1-(1-p)^{r+1}+p(1-p)^{r+1}(t-r) .
$$

Suppose that the event has a duration $X$ where $X$ is a random variable with distribution function $F_{X}(x)$. Then the event is detectable if and only if the sensor wakes up before the event terminates. Therefore, the detection probability $\alpha_{1}$ is given by

$$
\alpha_{1}=P\{W \leq X\}=\int_{0}^{\infty} P\{W \leq X \mid X=x\} d F_{X}(x)=\int_{0}^{\infty} F_{W}(x) d F_{X}(x) .
$$

Given that the event is detectable, the density function of the detection delay $D$ is

$$
f_{D}(t \mid W \leq X)=\int_{0}^{\infty} f_{D}(t \mid W \leq x) d F_{X}(x) .
$$

But $D=W$ under the condition $W \leq x$. Therefore,

$$
f_{D}(t \mid W \leq x)= \begin{cases}f_{W}(t) / F_{W}(x), & 0 \leq t \leq x \\ 0, & \text { otherwise }\end{cases}
$$

Substituting (23), into (22), we have

$$
f_{D}(t \mid W \leq X)=\int_{t}^{\infty} \frac{f_{W}(t)}{F_{W}(x)} d F_{X}(x) .
$$

\subsection{Multiple Sensors}

Following the same scenario, we consider the case that the location is monitored by a fixed number of $n$ sensors. Let $W_{1}, W_{2}, \ldots, W_{n}$ be the wakeup delays of 
these $n$ sensors, and $W=\min \left\{W_{1}, W_{2}, \ldots, W_{n}\right\}$ be the smallest wakeup delay. The cumulative distribution function of $W$ is given by

$$
\begin{aligned}
F_{W}^{(n)}(t) & =P\{W \leq t\} \\
& =P\{\text { At least one sensor wakes up by time } t\} \\
& =1-P\{\text { All } n \text { sensors are sleeping by time } t\} \\
& =1-\left[1-F_{W}(t)\right]^{n}
\end{aligned}
$$

under the assumption that all sensors are working independently.

Replacing $f_{W}(t)$ by $f_{W}^{(n)}(t)$ in (21) and (24), we can obtain the detection probability $\alpha_{n}$ and the conditional detection delay distribution $f_{D}^{(n)}(t \mid W \leq X)$ in a straightforward manner. If $X$ is deterministic, two special cases are worth noting:

(i) $X \rightarrow 0$. Taking limit we have

$$
\alpha_{n}=\lim _{x \rightarrow 0} \int_{0}^{x} F_{W}^{(n)}(t) d F_{X}(t)=\lim _{x \rightarrow 0} F_{W}^{(n)}(x)=1-(1-p)^{n}
$$

from (25), noting that $F_{W}(0)=p$. This is the case where events can only be detected at the instance of occurrence. Therefore, to detect the event, at least one sensor must be awake. The probability is simply $1-(1-p)^{n}$, the same as the formal derivation above.

(ii) $X \rightarrow \infty$. In this case, the event can always be detected. Therefore, only the detection delay matters. But this is just the wakeup delay $W$ with the distribution function given by (25). The expected detection delay is as follows:

$$
\begin{aligned}
E[D] & =\int_{0}^{\infty}\left[1-F_{W}^{(n)}(t)\right] d t \\
& =\int_{0}^{\infty}\left[(1-p)^{r+1}-p(1-p)^{r+1}(t-r)\right]^{n} d t \\
& =\sum_{r=0}^{\infty} \int_{r}^{r+1}\left[(1-p)^{r+1}-p(1-p)^{r+1}(t-r)\right]^{n} d t \\
& =\sum_{r=0}^{\infty} \frac{1-(1-p)^{n+1}}{(n+1) p}(1-p)^{n(r+1)} \\
& =\frac{(1-p)^{n}}{(n+1) p} \cdot \frac{1-(1-p)^{n+1}}{1-(1-p)^{n}} .
\end{aligned}
$$

If $p$ is small and $n$ is not too small, $(27)$ can be reduced to $E[D] \approx 1 / n p$. In other words, the expected detection delay is inversely proportional to $n$ and $p$.

\subsection{Random Number of Sensors}

Let the location be monitored by $N$ sensors and $N$ be a Poisson random variable with rate $\lambda$ [Kumar et al. 2004; Zhang and Hou 2004b]. Following the previous 
derivation, we have

$$
F_{W}^{(N)}(t)=\sum_{n=0}^{\infty} F_{W}^{(n)}(t) \frac{e^{-\lambda} \lambda^{n}}{n !}=1-e^{-\lambda F_{W}(t)} .
$$

Replacing $f_{W}(t)$ by $f_{W}^{(N)}(t)$ in $(21)$, we obtain the detection probability $\alpha_{N}$. The notation of detection delay is meaningful only under the condition that the location is monitored by at least one sensor, or $N \geq 1$. Including this condition, we obtain

$$
f_{D}(t \mid W \leq X, N \geq 1)=\int_{t}^{\infty} \frac{f_{W}(t)}{\left(1-e^{-\lambda}\right) F_{W}(x)} d F_{X}(x) .
$$

Again, for two special types of events where $X$ approaches to 0 and $\infty$, respectively, we have the following:

(i) $X \rightarrow 0$. We have

$$
\alpha_{N}=\lim _{x \rightarrow 0}\left(1-e^{-\lambda F_{W}(x)}\right)=1-e^{-\lambda p} .
$$

To detect this type of event, at least one sensor should be awake at the event occurrence time. Since the number of sensors is Poisson- distributed with rate $\lambda$, and each sensor has the probability $p$ to be awake, the probability is therefore $1-e^{\lambda p}$.

(ii) $X \rightarrow \infty$. The detection probability is

$$
\alpha_{N}=\lim _{x \rightarrow \infty}\left(1-e^{-\lambda F_{W}(x)}\right)=1-e^{-\lambda} .
$$

This is equal to the probability that the location is monitored by at least one sensor. Under this condition, the expected detection delay $D$ can be derived from (29) as

$$
E[D]=\frac{e^{-\lambda}}{1-e^{-\lambda}} \sum_{r=1}^{\infty}\left[\frac{1}{\lambda p(1-p)^{r}}\left(e^{\lambda(1-p)^{r}}-e^{\lambda(1-p)^{r+1}}\right)-1\right] .
$$

\section{PERFORMANCE EVALUATION}

\subsection{Coverage and Vulnerable Periods}

In Figure 5 we show analytical and simulation results of expected coverage periods of ARS for various $k$ values. For simulation, the wakeup times of sensors are recorded and the average coverage periods for various $k$ values are obtained from the same set of data. The simulation results shown in Figure 5 are the average values of 10 simulation runs, and $95 \%$ confidence intervals are shown for all the simulation points. We see that they match perfectly with the analytical results. To quote a design example, point A shows that, with $p=0.1$, the expected 1-coverage period is 1.87 slots. By increasing $p$ to 0.2 , the expected 1-coverage period can be extended to 4.16 slots, as shown by point B (i.e., an increase of $122 \%$ ). But for the 2-coverage case, the corresponding values are 0.68 and 1.16 slots, as shown by points $\mathrm{C}$ and $\mathrm{D}$, respectively, an increase of $70 \%$. Together with the 3 -coverage results, we see that increasing $p$ does not 


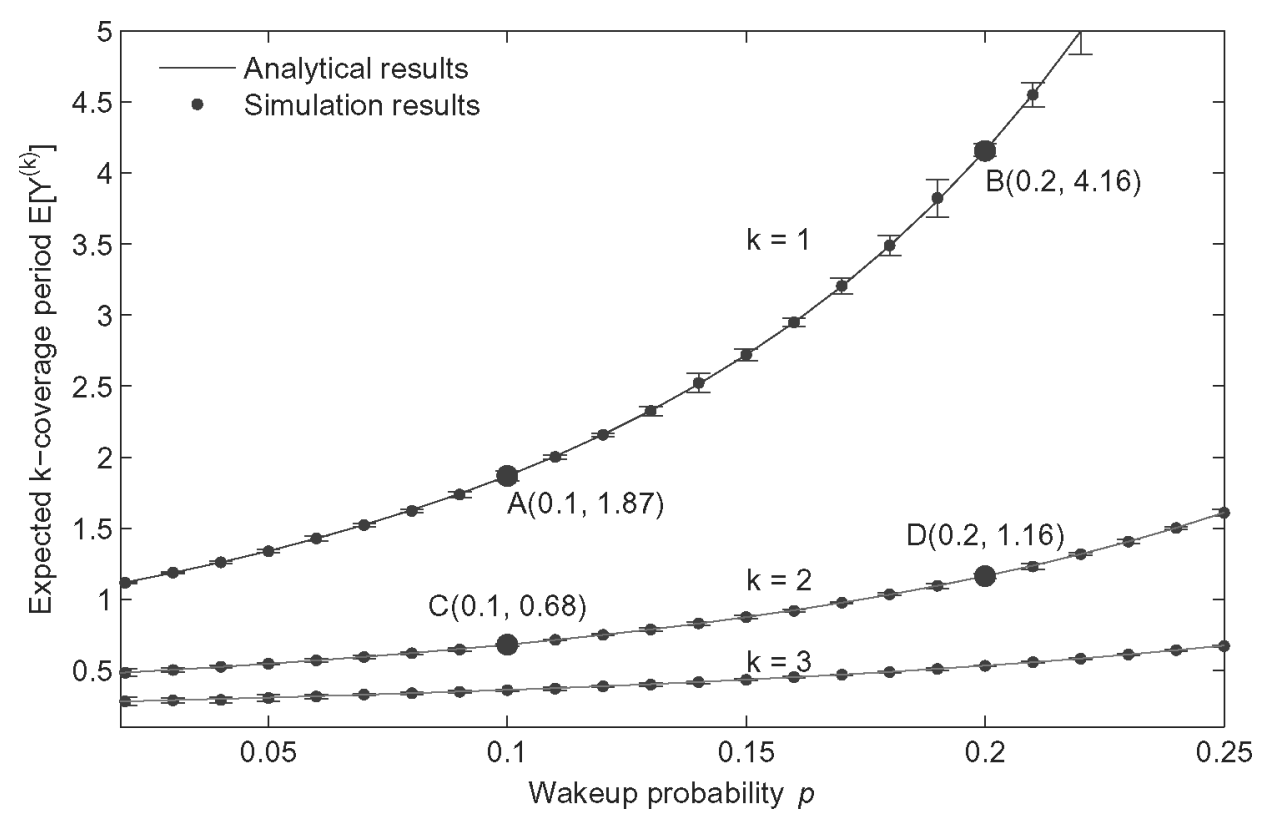

Fig. 5. Expected coverage period $E\left[Y^{(k)}\right]$ versus wakeup probability $p$, and $n=10$.

help much for higher-dimensional coverage. From the same set of data, we obtain the expected vulnerable periods of ARS for various $k$ values and show the results in Figure 6. We see that in the lower duty-cycle regime, the vulnerable period decreases quickly comparing with the increase of coverage period given the same increment of wakeup probability.

\subsection{Detection Delay Distribution}

Figure 7 shows the distribution function of detection delay for the multiplesensors case with fixed wakeup probability $p=0.1$. The $95 \%$ confidence intervals are shown for all the simulation points. We see a perfect match between the analytical and simulation results. For the random sensors case, the event is detectable only with probability $1-e^{-\lambda}$. This is shown in Figure 8 , where we plot the distribution function of the detection delay for $p=0.1 ; X \rightarrow \infty$ and $\lambda$ varies from 3 to 9 . When $D \rightarrow \infty$, we see that the asymptomatic values of $1-e^{-\lambda}$ are approached.

Wakeup probability is another important factor in detection delay. In Figure 9 we show the detection delay distribution for the multiple-sensors case with $n=5$ and wakeup probability varying from 0.1 to 0.5 . We can see that increasing the wakeup probability can reduce the detection delay significantly. For $p=$ 0.1 , only about $80 \%$ of the events can be detected within 1 time unit, but if the wakeup probability is increased to 0.3 and 0.5 , the detection percentage approaches $98 \%$ and $100 \%$, respectively, for the same detection delay. Similar observations can be made from Figure 10, which shows the same result for the random sensors case. We can see the tradeoff between energy efficiency and surveillance performance from these figures. To detect events with a smaller 
$\begin{array}{lll}\text { Article } 15 / 16 & \text { C. Q. Hua and T.-S. Yum }\end{array}$

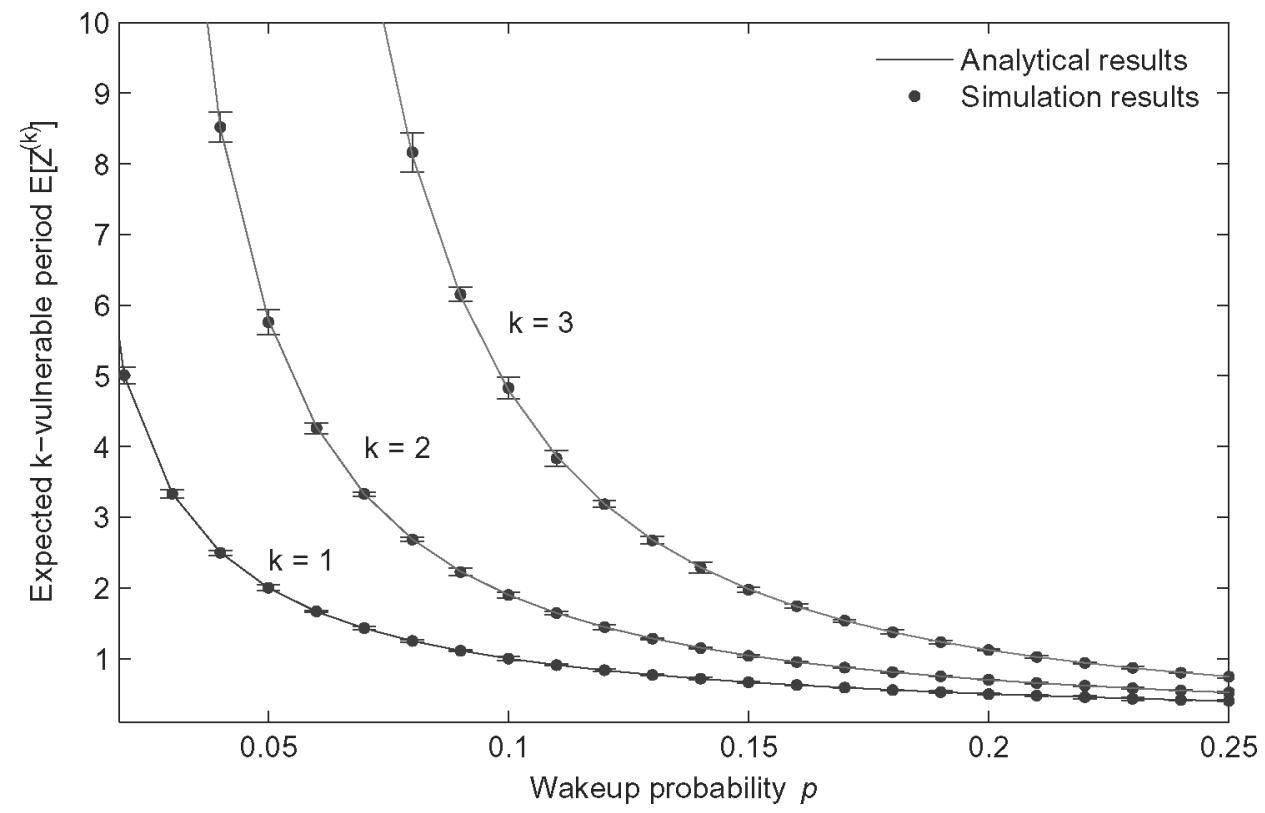

Fig. 6. Expected vulnerable period $E\left[Z^{(k)}\right]$ versus wakeup probability $p$, and $n=10$.

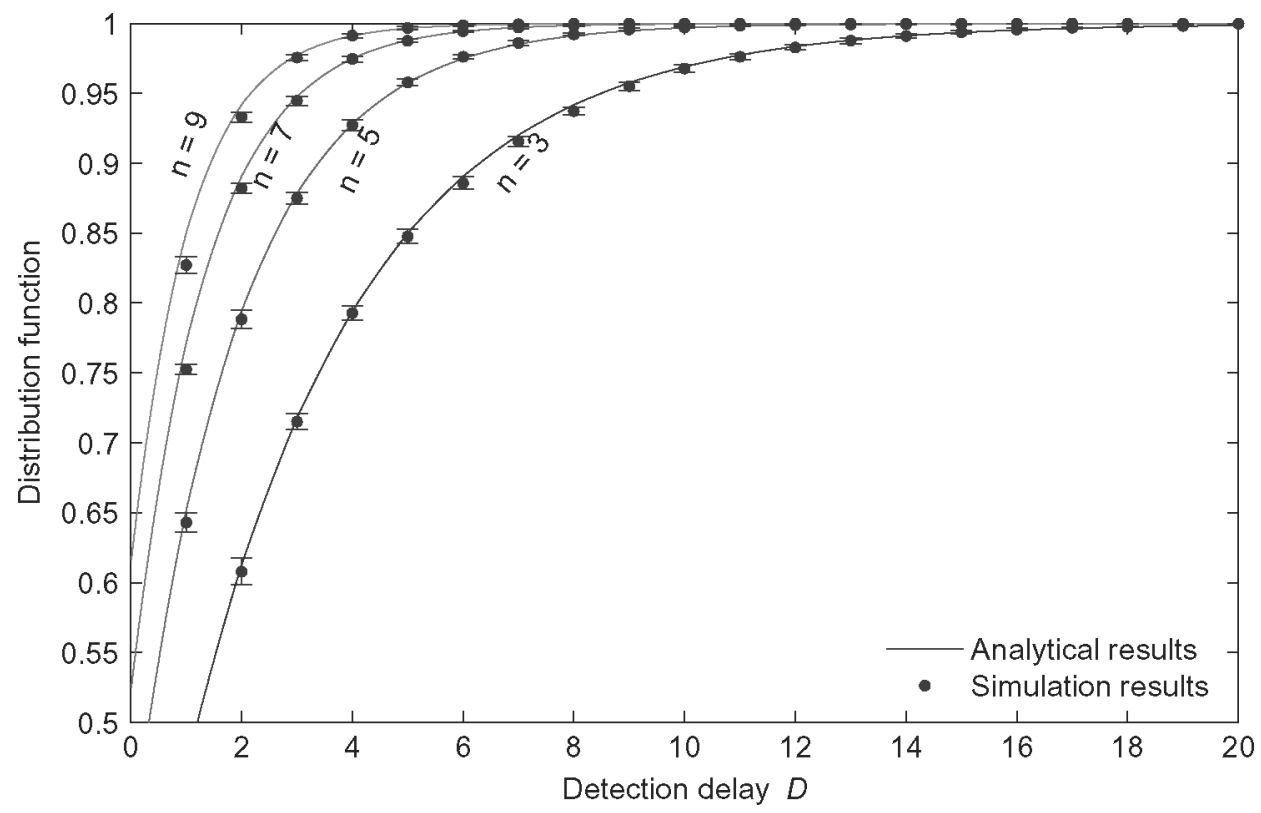

Fig. 7. Distribution function of detection delay: multiple-sensors case, $X \rightarrow \infty$ and $p=0.1$. 


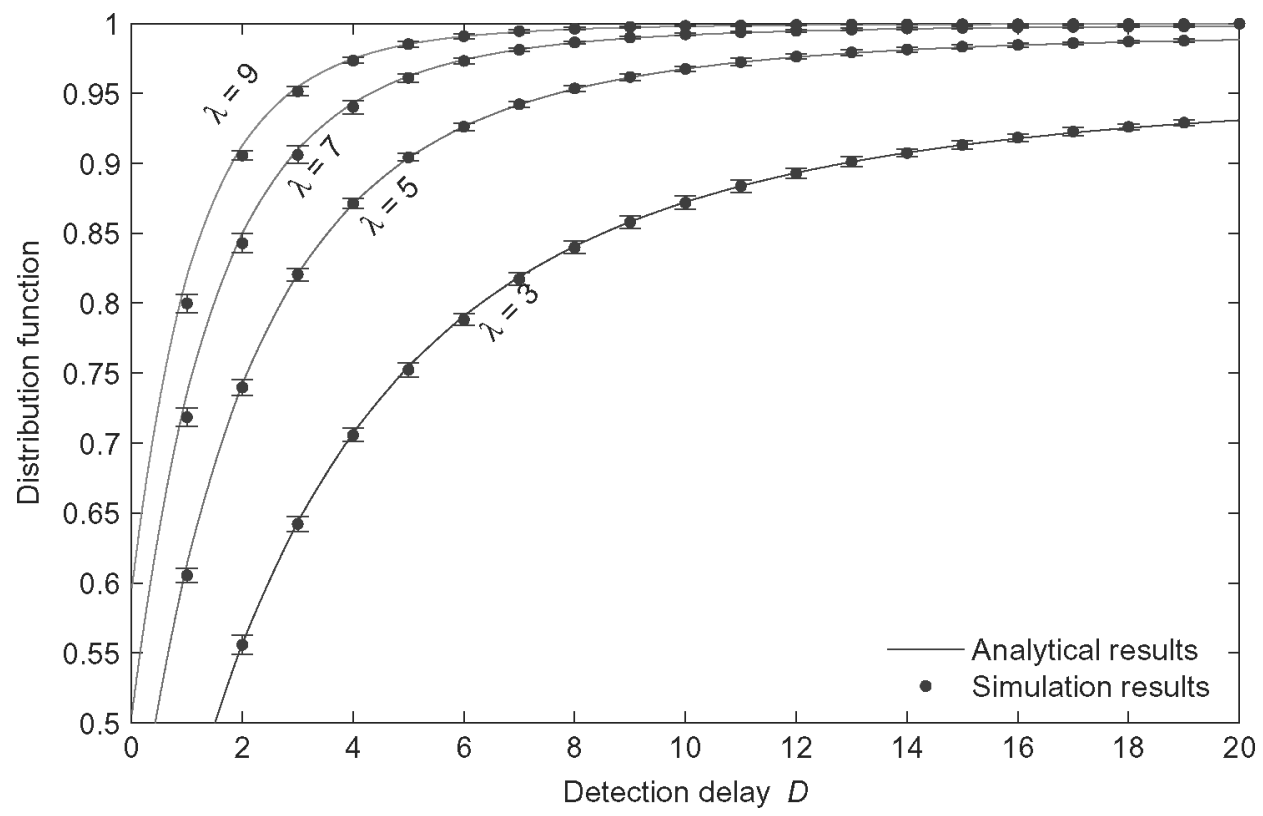

Fig. 8. Distribution function of detection delay: random sensors case, $X \rightarrow \infty$ and $p=0.1$.

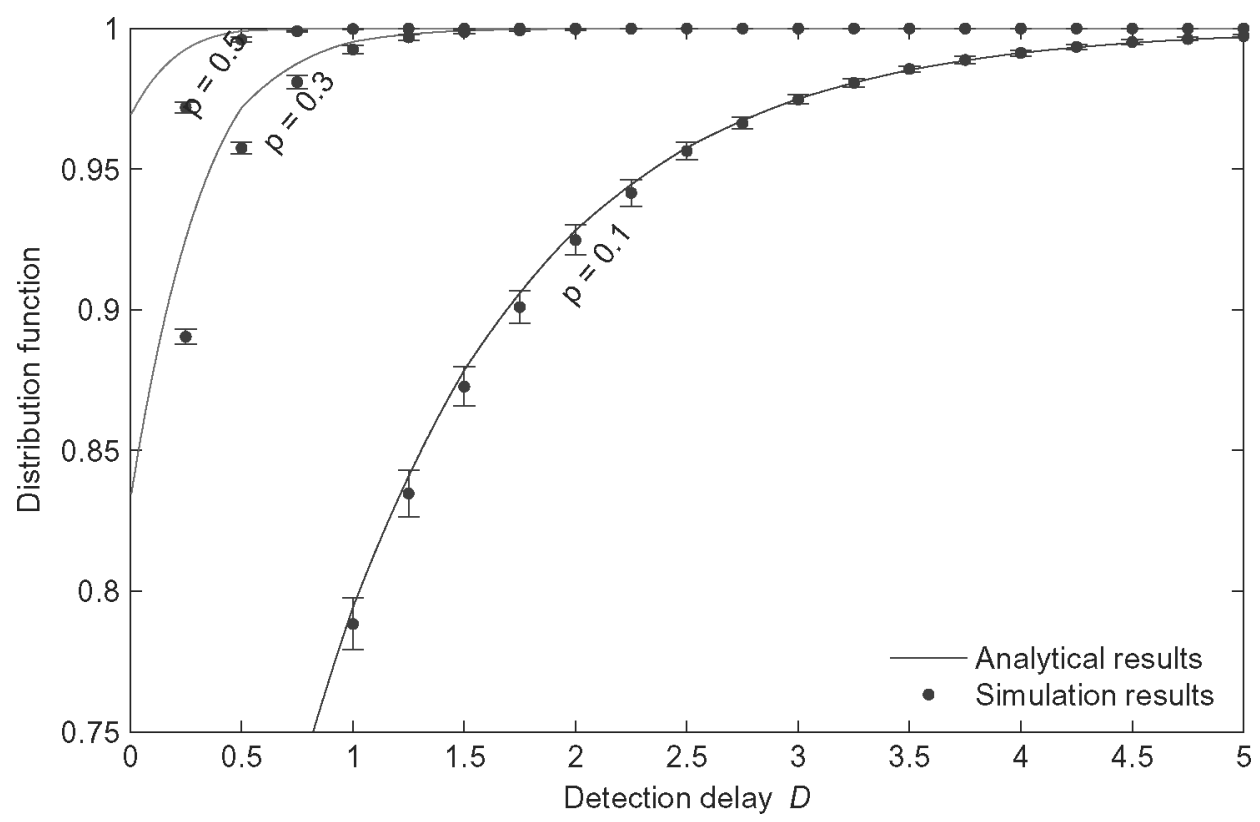

Fig. 9. Distribution function of detection delay: multiple-sensors case, $X \rightarrow \infty$ and $n=5$. 


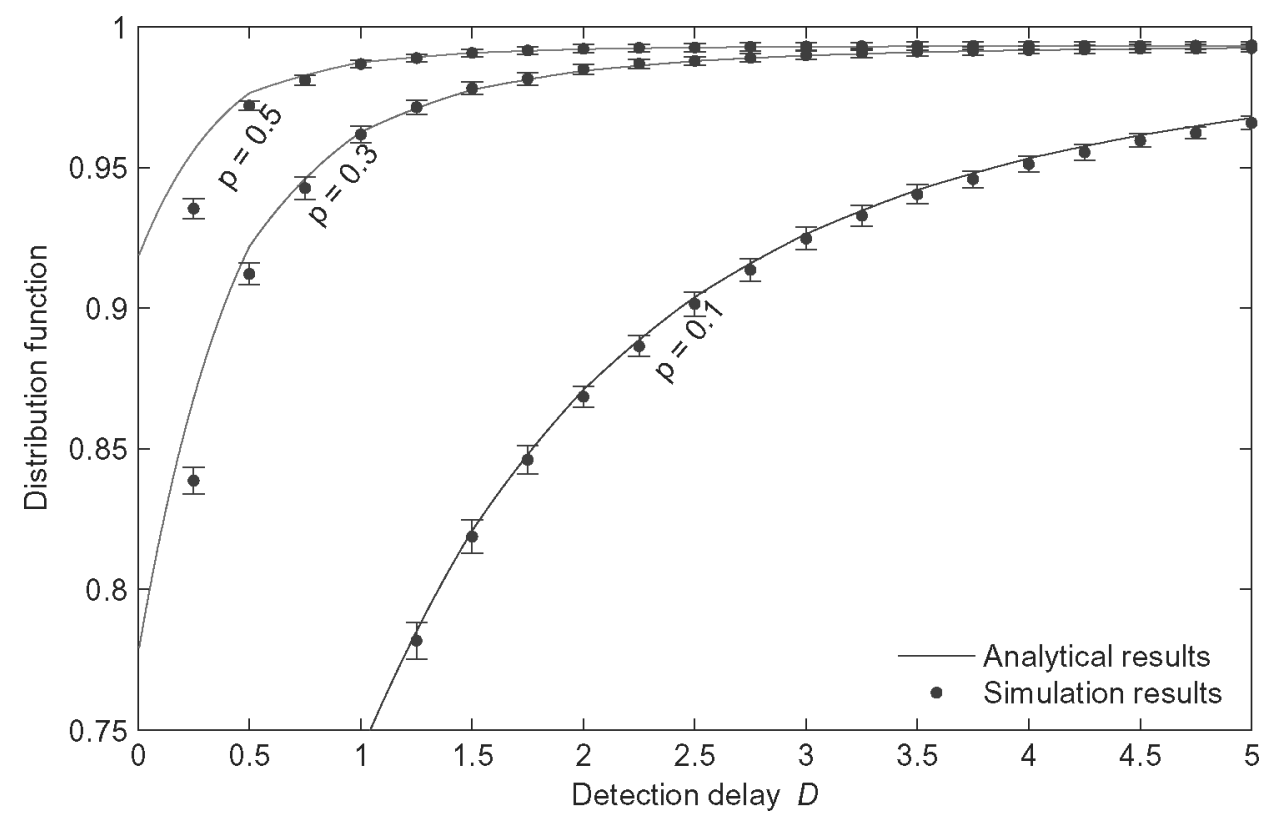

Fig. 10. Distribution function of detection delay: random sensors case, $X \rightarrow \infty$ and $\lambda=5$.

delay, one has to put the sensors into a high duty cycle and wake up more often, thus increasing the energy usage and reducing the node lifetime. Therefore, the node density and wakeup probability should be designed according to the application requirements.

\subsection{Impact of Clock Drifting}

In our model, we assume that time offsets of different nodes are uniformly distributed and remain constant with respect to each other. However, due to the absence of time synchronization among sensor nodes, the time offsets may change over time due to clock drifting. In this section, we study the impact of clock drifting on the detection delay. We assume that each node $i$ has a clock drifting rate $\delta_{i}, 0 \leq \delta_{i} \leq 1$, that defines the percentage of time unit the clock will drift within each time slot. All drifting rates $\delta_{i}$ s are i.i.d. random variables in $\left(0, \delta_{\max }\right)$, where $\delta_{\max }$ defines the maximum possible drifting rate. We run the simulations for two drifting scenarios, the slow drifting case $\left(\delta_{\max }=0.01\right)$ and the fast drifting case $\left(\delta_{\max }=0.1\right)$. The results are compared with the nondrifting case. In Figure 11, we show the comparison of the expected detection delay with multiple sensors $(n=5,10)$. We can see that clock drifting has no significant impact on the detection performance as the average detection delays of all these cases are very close for different wakeup probabilities and node densities. Similar results can be observed for random sensors case, as shown in Figure 12. These figures suggest that our results can be a good approximation for clock drifting case. 


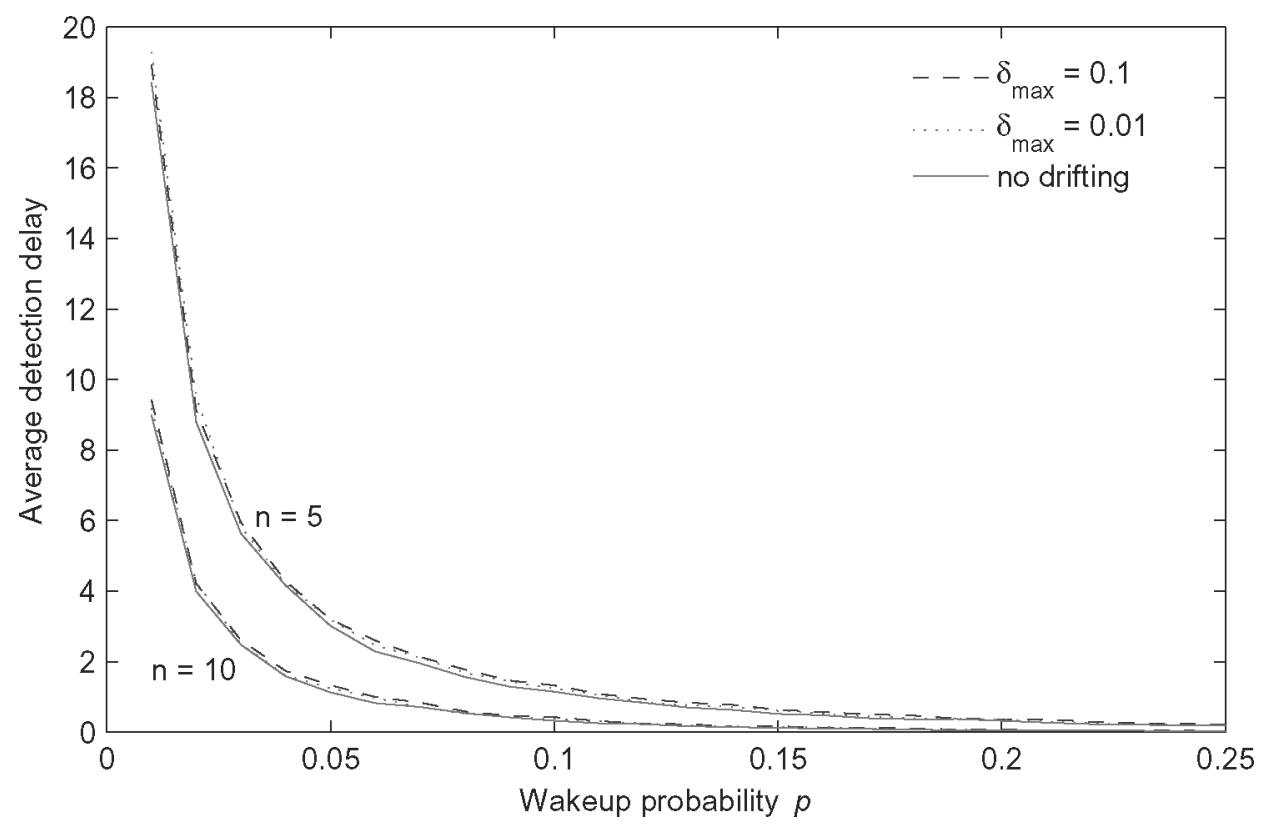

Fig. 11. Comparison of detection delay with clock drifting: multiple-sensors case.

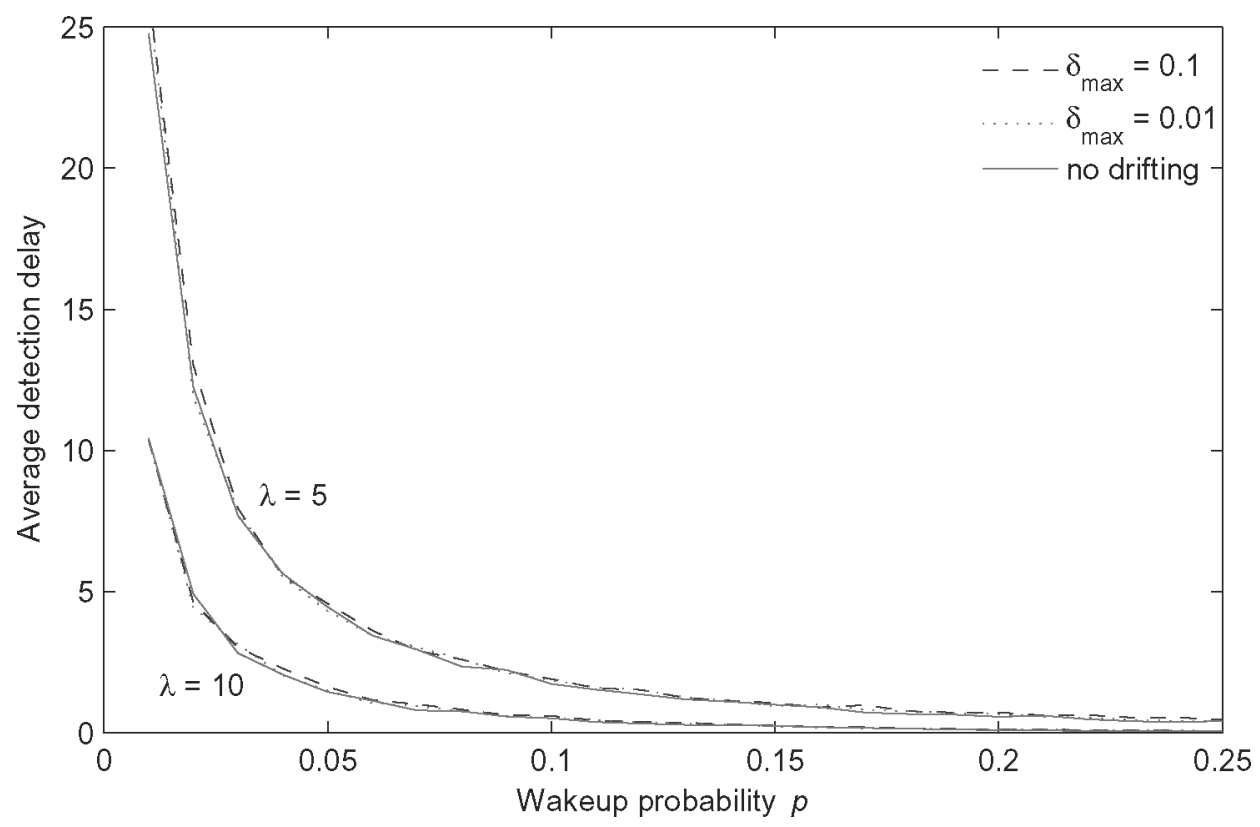

Fig. 12. Comparison of detection delay with clock drifting: random sensors case. 


\subsection{Comparison of ARS, RIS, and RDS}

In this section, we compare the performance of ARS with RIS [Kumar et al. 2004] and RDS [Cao et al. 2005b].

RIS is a synchronous random sleeping scheme. Using the same techniques as in Section 5, we first consider the case where a location is monitored by $n$ sensors, and these sensors are synchronized and working independently. Following the derivation of (19), the PDF of the wakeup delay $W$ is given by

$$
f_{W}^{(n)}(t)=\left(1-(1-p)^{n}\right)(1-p)^{n(r+1)}
$$

and the corresponding distribution function is

$$
F_{W}^{(n)}(t)=1-(1-p)^{n(r+1)}+\left(1-(1-p)^{n}\right)(1-p)^{n(r+1)}(t-r) .
$$

For event with duration $X \rightarrow \infty$, the detection probability is always 1 and so we only need to consider the expected detection delay, which is given by

$$
E[D]=\int_{0}^{\infty}\left[1-F_{W}^{(n)}(t)\right] d t=\frac{1+(1-p)^{n}}{2} \cdot \frac{(1-p)^{n}}{1-(1-p)^{n}} .
$$

For the random nodes case, the distribution function of the wakeup delay is

$$
\begin{aligned}
F_{W}^{(N)}(t) & =\sum_{n=0}^{\infty} F_{W}^{(n)}(t) \frac{e^{-\lambda} \lambda^{n}}{n !} \\
& =1-e^{-\lambda}\left[e^{\lambda(1-p)^{r+1}}-e^{\lambda(1-p)^{r+1}}(t-r)+e^{\lambda(1-p)^{r+2}}(t-r)\right] .
\end{aligned}
$$

where $F_{W}^{(n)}(t)$ is given by (34). Similarly to (32), we can obtain the conditional expectation for the detection delay $D$ as

$$
E[D]=\frac{e^{-\lambda}}{1-e^{-\lambda}} \sum_{r=1}^{\infty}\left[\frac{1}{2}\left(e^{\lambda(1-p)^{r}}+e^{\lambda(1-p)^{r+1}}\right)-1\right] .
$$

RDS is another asynchronous sleeping scheme proposed for surveillance application [Cao et al. 2005b]. In RDS, all nodes have the same scheduling period $T$ and duty cycle $p, 0 \leq p \leq 1$. Each node independently wakes up at some random time $t_{\text {start }} \in[0, T]$ and remains awake for a time period $p T$, and goes back to sleep until the next time slot. For a fair comparison of RDS and ARS, the scheduling period $T$ should be scaled to $T=1 / p$; thus each node wakes up only 1 time unit in every $1 / p$ time units. It is straightforward to obtain the detection delay distribution of RDS from the results of Cao et al. [2005b] by substituting $T$ with $1 / p$. For the multiple-sensors case, the expected detection delay is given by

$$
E[D]=\frac{(1-p)^{n+1}}{(n+1) p} .
$$

For the random sensors case, the expected detection delay is given by

$$
E[D]=\frac{e^{-\lambda p}}{\lambda p\left(1-e^{-\lambda}\right)} .
$$




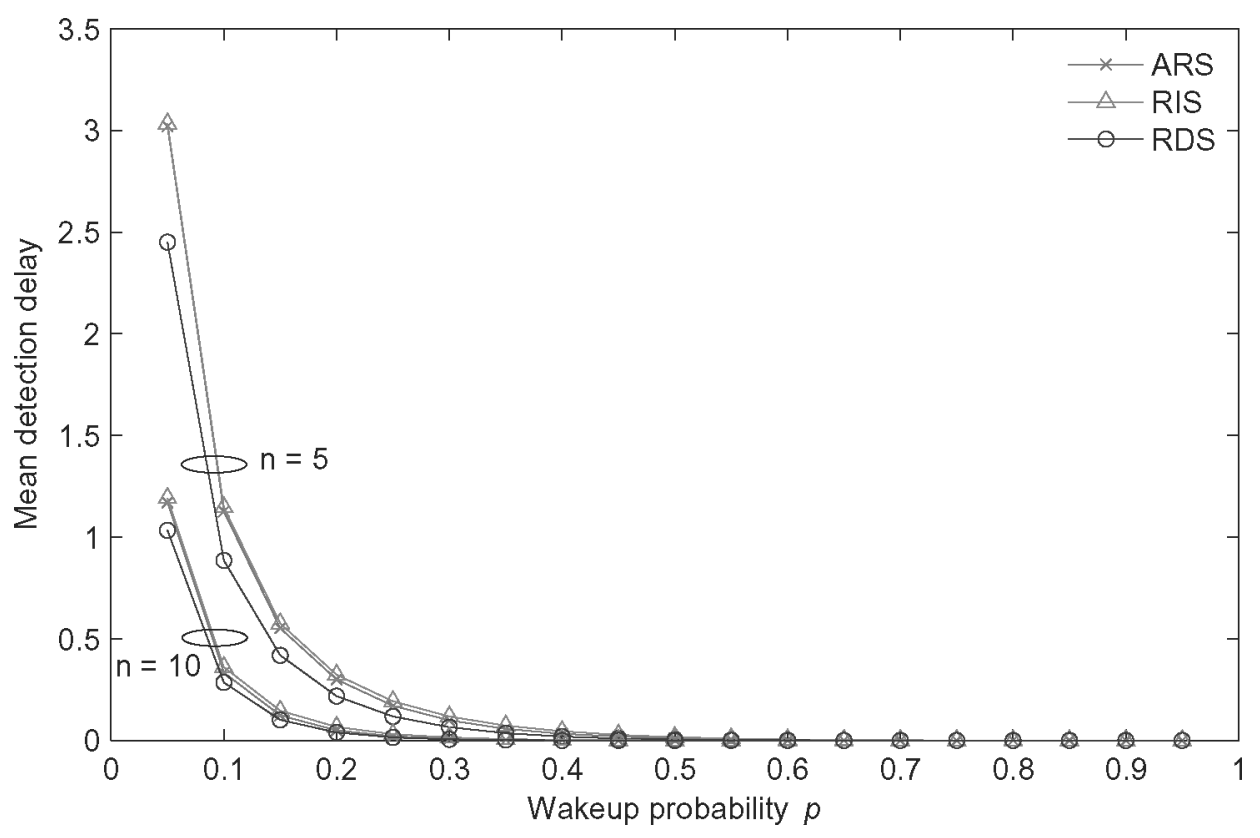

Fig. 13. Comparison of ARS, RIS, and RDS: multiple-sensors case.

In Figure 13, we compare the expected detection delay of ARS with RIS and RDS schemes for the multiple-sensors case. We see that the performance of ARS and RIS is quite close. The detection delay obtained by these schemes is slightly larger than that of RDS in the lower duty-cycle regime (with smaller wakeup probability). However, the performance gap diminishes with the increase of wakeup probability. For the random sensors case, we see different results in Figure 14. In the lower duty-cycle regime $(p \leq 0.1)$, we find that RDS leads to a larger detection delay than the ARS and RIS schemes, which is especially true for smaller node density $(\lambda=5)$. However, in the higher duty-cycle regime, the result is similar to the multiple-sensors case. Therefore, if a sensor network is required to work in the lower duty-cycle regime for saving energy consumption, ARS is more desirable than RDS since it can offer better detection delay with the same energy efficiency.

\section{CONCLUSIONS}

In this article we have derived the closed-form expressions for the expected $k$-coverage and $k$-vulnerable periods of ARS. The detection probability and detection delay of ARS were derived for surveillance-type applications. We have illustrated the performance of ARS with extensive numerical and simulation results and compared it with other synchronous and asynchronous sleeping scheduling schemes. Design examples were provided to demonstrate how to use our analytical results to choose system and protocol parameters according to the application requirements. It is interesting to extend the analysis of this article to the case where sensors have heterogeneous wakeup probabilities, which, however, is beyond the scope of this article. 
$\begin{array}{lll}\text { Article } 15 / 22 & \text { C. Q. Hua and T.-S. Yum }\end{array}$

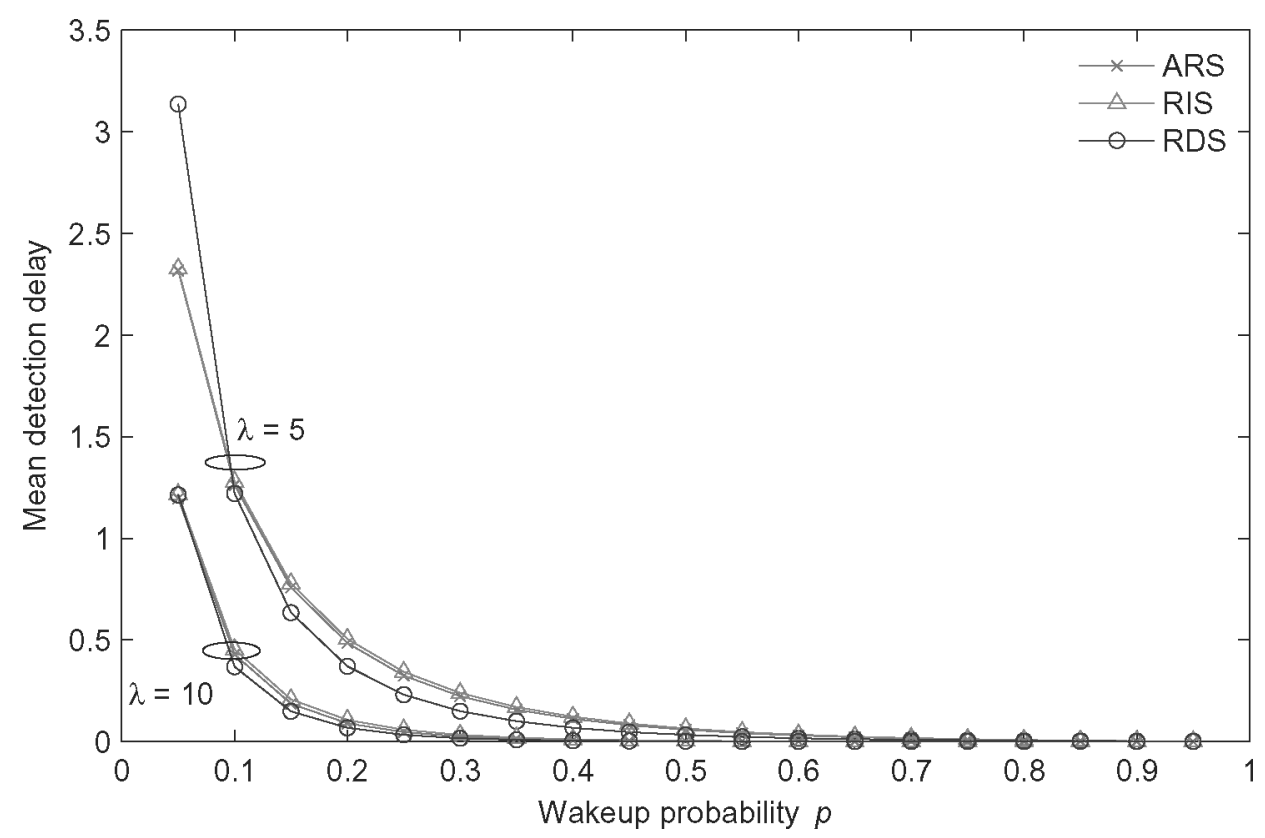

Fig. 14. Comparison of ARS, RIS, and RDS: random sensors case.

\section{APPENDIX}

\section{A. Derivation of $B^{*}(s)$}

For each $j$, we have the density function of $V_{j}$ 's from (1). Applying the Laplace transform to all $V_{j}$ 's to obtain $B_{j}^{*}(s)$, we have

$$
\begin{aligned}
B^{*}(s)= & \sum_{j=1}^{n-1} p(1-p)^{j-1} B_{j}^{*}(s) \\
= & \sum_{j=1}^{n-1} p(1-p)^{j-1} \int_{0}^{1} \frac{\Gamma(n)}{\Gamma(n-j) \Gamma(j)} y^{j-1}(1-y)^{n-1-j} e^{-s y} d y \\
= & \int_{0}^{1} e^{-s y}\left(\sum_{j=1}^{n-1} p(1-p)^{j-1} \frac{\Gamma(n)}{\Gamma(n-j) \Gamma(j)} y^{j-1}(1-y)^{n-1-j}\right) d y \\
= & \int_{0}^{1} e^{-s y}(n-1) p(1-p y)^{n-2} d y \\
= & \frac{(n-1) p^{n-1}}{s^{n-1}}\left\{\left[\left(\frac{s}{p}\right)^{n-2}-(n-2)\left(\frac{s}{p}\right)^{n-3} \cdots+(-1)^{n-2}(n-2) !\right]\right. \\
& \left.-e^{-s}\left[\left(\frac{s(1-p)}{p}\right)^{n-2}-(n-2)\left(\frac{s(1-p)}{p}\right)^{n-3} \cdots+(-1)^{n-2}(n-2) !\right]\right\} .
\end{aligned}
$$




\section{B. Derivation of Equation (10)}

In (8), subtracting the $i-1$ th equation from the $i$ th equation, we have

$$
E\left[Y_{i}^{(2)}\right]-E\left[Y_{i-1}^{(2)}\right]=\frac{(1-p)^{i-1}}{n}+p(1-p)^{i-1} E\left[Y_{n-i}^{(2)}\right], \quad i=2, \ldots, n-1 .
$$

Multiplying the $i-1$ th equation of (B-1) by $(1-p)$ and subtracting it from the $i$ th equation, we obtain

$$
\begin{aligned}
E & {\left[Y_{i}^{(2)}\right]-E\left[Y_{i-1}^{(2)}\right]-(1-p)\left(E\left[Y_{i-1}^{(2)}\right]-E\left[Y_{i-2}^{(2)}\right]\right) } \\
& =p(1-p)^{i-1}\left(E\left[Y_{n-i}^{(2)}\right]-E\left[Y_{n-i+1}^{(2)}\right]\right) \\
& =-p(1-p)^{i-1}\left(\frac{(1-p)^{n-i}}{n}+p(1-p)^{n-i} E\left[Y_{i-1}^{(2)}\right]\right) \\
& =-\frac{p(1-p)^{n-1}}{n}-p^{2}(1-p)^{n-1} E\left[Y_{i-1}^{(2)}\right], i=2, \ldots, n-1,
\end{aligned}
$$

where the second equality is obtained by substituting the $n-i$ equation of (B-1).

Solving for $E\left[Y_{i}^{(2)}\right]$ from (B-2), we obtain a recursive equation for $E\left[Y_{i}^{(2)}\right]$ as

$$
E\left[Y_{i}^{(2)}\right]=a E\left[Y_{i-1}^{(2)}\right]-(1-p) E\left[Y_{i-2}^{(2)}\right]-b, \quad i=2, \ldots, n-1,
$$

where $a=2-p-p^{2}(1-p)^{n-1}$ and $b=p(1-p)^{n-1} / n$.

Recalling from (8), we have

$$
E\left[Y_{1}^{(2)}\right]=\frac{1}{n}+p E\left[Y_{n-1}^{(2)}\right] .
$$

Summing up (B-4) and (B-3) for $i=2, \cdots, n-1$ we have

$$
\begin{aligned}
\sum_{i=1}^{n-1} E\left[Y_{i}^{(2)}\right]= & a \sum_{i=1}^{n-2} E\left[Y_{i}^{(2)}\right]-(1-p) \sum_{i=1}^{n-3} E\left[Y_{i}^{(2)}\right]-(n-2) b+\frac{1}{n}+p E\left[Y_{n-1}^{(2)}\right] \\
= & a \sum_{i=1}^{n-1} E\left[Y_{i}^{(2)}\right]-(1-p) \sum_{i=1}^{n-1} E\left[Y_{i}^{(2)}\right]-(n-2) b+\frac{1}{n}+p E\left[Y_{n-1}^{(2)}\right] \\
& -a E\left[Y_{n-1}^{(2)}\right]+(1-p)\left(E\left[Y_{n-1}^{(2)}\right]+E\left[Y_{n-2}^{(2)}\right]\right) .
\end{aligned}
$$

Switching the first two terms of the right-hand side of (B-5) to the left-hand side, we have

$$
(2-p-a) \sum_{i=1}^{n-1} E\left[Y_{i}^{(2)}\right]=(1-a) E\left[Y_{n-1}^{(2)}\right]+(1-p) E\left[Y_{n-2}^{(2)}\right]-(n-2) b+\frac{1}{n} .
$$

From (B-4) and (B-1) we know that $p E\left[Y_{n-1}^{(2)}\right]=E\left[Y_{1}^{(2)}\right]-1 / n$ and $p(1-$ p) $E\left[Y_{n-2}^{(2)}\right]=E\left[Y_{2}^{(2)}\right]-E\left[Y_{1}^{(2)}\right]-(1-p) / n$; substituting them into (B-6) we 
$\begin{array}{lll}\text { Article } 15 / 24 & \text { C. Q. Hua and T.-S. Yum }\end{array}$

have

$$
\begin{aligned}
(2-p-a) \sum_{i=1}^{n-1} E\left[Y_{i}^{(2)}\right]= & \frac{1-a}{p}\left(E\left[Y_{1}^{(2)}\right]-\frac{1}{n}\right) \\
& +\frac{1}{p}\left(E\left[Y_{2}^{(2)}\right]-E\left[Y_{1}^{(2)}\right]-\frac{1-p}{n}\right) . \\
& -(n-2) b+\frac{1}{n}=\frac{E\left[Y_{2}^{(2)}\right]-a E\left[Y_{1}^{(2)}\right]}{p} \\
& +\frac{1+p(1-p)^{n-1}-n p(1-p)^{n-1}}{n} .
\end{aligned}
$$

Note $2-p-a=p^{2}(1-p)^{n-1}$. From (B-3) we know $E\left[Y_{2}^{(2)}\right]-a E\left[Y_{1}^{(2)}\right]=-b$; therefore the above equation can be simplified as

$$
\sum_{i=1}^{n-1} E\left[Y_{i}^{(2)}\right]=\frac{1-(1-p)^{n}-n p(1-p)^{n-1}}{n p^{2}(1-p)^{n-1}} .
$$

Dividing (B-8) by $\left(\begin{array}{l}n \\ 1\end{array}\right)$ gives (10).

\section{REFERENCES}

Aspnes, J., GoldenberG, D., ANd YAnG, Y. R. 2004. On the computational complexity of sensor network localization. In Proceedings of the First International Workshop on Algorithmic Aspects of Wireless Sensor Networks.

Cao, Q., Abdelzaher, T., He, T., and Stankovic, J. 2005a. Towards optimal sleep scheduling in sensor networks for rare-event detection. In Proceedings of the 4th International Symposium on Information Processing in Sensor Networks. IEEE Press, Los Alomitos, CA, 4.

Cao, Q., Yan, T., Stankovic, J., ANd Abdelzaher, T. 2005b. Analysis of target detection performance for wireless sensor networks. In International Conference on Distributed Computing in Sensor Networks (DCOSS).

Crossbow. 2004. Power management and batteries. Available online at http://www.xbow.com/ Support/appnotes.htm.

Deng, J., Han, Y. S., Heinzelman, W. B., and Varshney, P. K. 2005. Balanced-energy sleep scheduling scheme for high density cluster-based sensor networks. Elsevier Comput. Commun. J. (Special Issue on ASWN '04) 28, 14 (Sept.), 1631-1642.

ELson, J., Girod, L., AND Estrin, D. 2002. Fine-grained network time synchronization using reference broadcasts. SIGOPS Oper. Syst. Rev. 36, SI, 147-163.

Gui, C. And Mohapatra, P. 2004. Power conservation and quality of surveillance in target tracking sensor networks. In Proceedings of the 10th Annual International Conference on Mobile Computing and Networking. (Philadelphia, PA). 129-143.

He, T., Krishnamurthy, S., Stankovic, J. A., Abdelzaher, T., Luo, L., Stoleru, R., Yan, T., Gu, L., Hui, J., AND KROGH, B. 2004. Energy-efficient surveillance system using wireless sensor networks. In Proceedings of the 2nd International Conference on Mobile Systems, Applications, and Services (Boston, MA). ACM Press, New York, NY, 270-283.

Hsin, C.-F. AND Liv, M. 2004. Network coverage using low duty-cycled sensors: random \& coordinated sleep algorithms. In Proceedings of IPSN'04 (Berkeley, CA). 433-442.

Kumar, S., LAI, T. H., AND BALOGH, J. 2004. On k-coverage in a mostly sleeping sensor network. In Proceedings of MobiCom'04 (Philadelphia, PA). 144-158.

Li, X.-Y., WAN, P.-J., AND FRIEder, O. 2003. Coverage in wireless ad hoc sensor networks. IEEE Trans. Comput. 52, 6, 753-763.

Liv, B. AND Towsley, D. 2004. A study of the coverage of large-scale sensor networks. In Proceedings of MASS '04.

ACM Transactions on Sensor Networks, Vol. 3, No. 3, Article 15, Publication date: August 2007. 
Mathai, A. 1999. An Introduction to Geometrical Probability: Distributional Aspects with Applications. Gordon and Breach, Amsterdam, The Netherlands.

Moscibroda, T. and Wattenhofer, R. 2005. Maximizing the lifetime of dominating sets. In Proceedings of the 5th International Workshop on Algorithms for Wireless, Mobile, Ad Hoc and Sensor Networks (WMAN).

Paruchuri, V., Basavaraju, S., Durresi, A., Kannan, R., and Iyengar, S. $2004 . \quad$ Random asynchronous wakeup protocol for sensor networks. In Proceedings of the First International Conference on Broadband Networks. 710-717.

Ross, S. M. 1996. Stochastic Processes. Wiley, New York, NY.

Shakkottai, S., SRIKant, R., ANd Shroff, N. 2003. Unreliable sensor grids: Coverage, connectivity and diameter. In Proceedings of Infocom'03. Vol. 2. 1073-1083.

Tian, D. ANd Georganas, N. D. 2002. A coverage-preserving node scheduling scheme for large wireless sensor networks. In Proceedings of the 1st ACM International Workshop on Wireless Sensor Networks and Applications (WSNA'02, Atlanta, GA). 32-41.

WANG, L. AND XIAO, Y. Oct. 2005. Energy saving mechanisms in sensor networks. In Proceedings of IEEE Broadnets 2005.

Wang, X., Xing, G., Zhang, Y., Lu, C., Pless, R., And Gill, C. $2003 . \quad$ Integrated coverage and connectivity configuration in wireless sensor networks. In Proceedings of the 1st International Conference on Embedded Networked Sensor Systems. (Los Angeles, CA) ACM Press, New York, NY, $28-39$.

Yan, T., He, T., and Stankovic, J. A. 2003. Differentiated surveillance for sensor networks. In Proceedings of the 1st International Conference on Embedded Networked Sensor Systems. (Los Angeles, CA). ACM Press, New York, NY, 51-62.

Zhang, H. AND HoU, J. 2004a. Maintaining sensing coverage and connectivity in large sensor networks. In Proceedings of the NSF International Workshop on Theoretical and Algorithmic Aspects of Sensor, Ad HocWireless, and Peer-to-Peer Networks. Invited paper.

ZHANG, H. AND Hou, J. 2004b. On deriving the upper bound of $\alpha$-lifetime for large sensor networks. In Proceedings of MobiHoc'04 (Roppongi Hills, Tokyo, Japan). 121-132.

Zheng, R., Hou, J. C., AND SHA, L. 2003. Asynchronous wakeup for ad hoc networks. In Proceedings of the 4th ACM International Symposium on Mobile Ad Hoc Networking \& Computing. (Annapolis, MD). ACM Press, New York, NY, 35-45.

Received March 2006; revised August 2006, November 2006; accepted December 2006 